X-RAY OBSERVATIONS OF OBJECTS AT COSMOLOGICAL DISTANCES FROM THE "EINSTEIN" OBSERVATORY.

Riccardo Giacconi

Harvard/Smithsonian Center for Astrophysics

Cambridge, Massachusetts 02138

\title{
INTRODUCTION
}

The launch of the Einstein X-ray Observatory on November 13, 1978 has brought about a qualitative change in the field of X-ray astronomy. A fairly detailed description of the Observatory has appeared in print (Giacconi et al, 1979a) and therefore will not be given here.

However, a summary of the relevant instrument parameter's is useful. Figure 1 shows a schematic representation of the Observatory which was constructed under the management control of Marshall Space Flight Center of National Aeronautics and Space Administration. TRW, Inc. was responsible for the spacecraft and American Science \& Engineering, Inc. for much of the instrumentation. The X-ray telescope, built by Perkin-Elmer, has a 0.6 meter aperture with a focal plane scale of 1 arc $\mathrm{min} / \mathrm{mm}$. It consists of 4 nested Wolter-type I paraboloid/hyperboloid grazing incidence mirrors. The telescope effective area is $\sim 400 \mathrm{~cm}^{2}$ at $0.25 \mathrm{keV}$ and $\sim 30 \mathrm{~cm}^{2}$ at $4 \mathrm{keV}$.

The detectors are on a lazy-Susan arrangement which permits each instrument to be placed in the telescope focus. There are two types of imaging detectors: (a) an imaging proportional counter (IPC) with large field of view $\left(\sim 1^{\circ} \times 1^{\circ}\right)$, moderate angular resolution $(\sim 2$ arc $\mathrm{min})$, and broadband spectral resolution from 0.25 to $4.0 \mathrm{keV}$, and (b) a high resolution imaging detector (HRI) which is limited by the telescope point response function to about 3 arc seconds resolution, with no spectral resolution. There are two types of spectrometers: (a) a non-dispersive $\mathrm{Si}(\mathrm{Li})$ crystal spectrometer, cooled to about $100 \mathrm{k}$, with high sensitivity and $150 \mathrm{eV}$ resolution in the 0.8 to $3.5 \mathrm{keV}$ range, and (b) a high resolution, but low sensitivity, Bragg Crystal Spectrometer. The imaging detectors were developed by the Center for Astrophysics, while the spectrometers were developed by Goddard Space Flight Center and Massachusetts Institute of Technology respectively. 


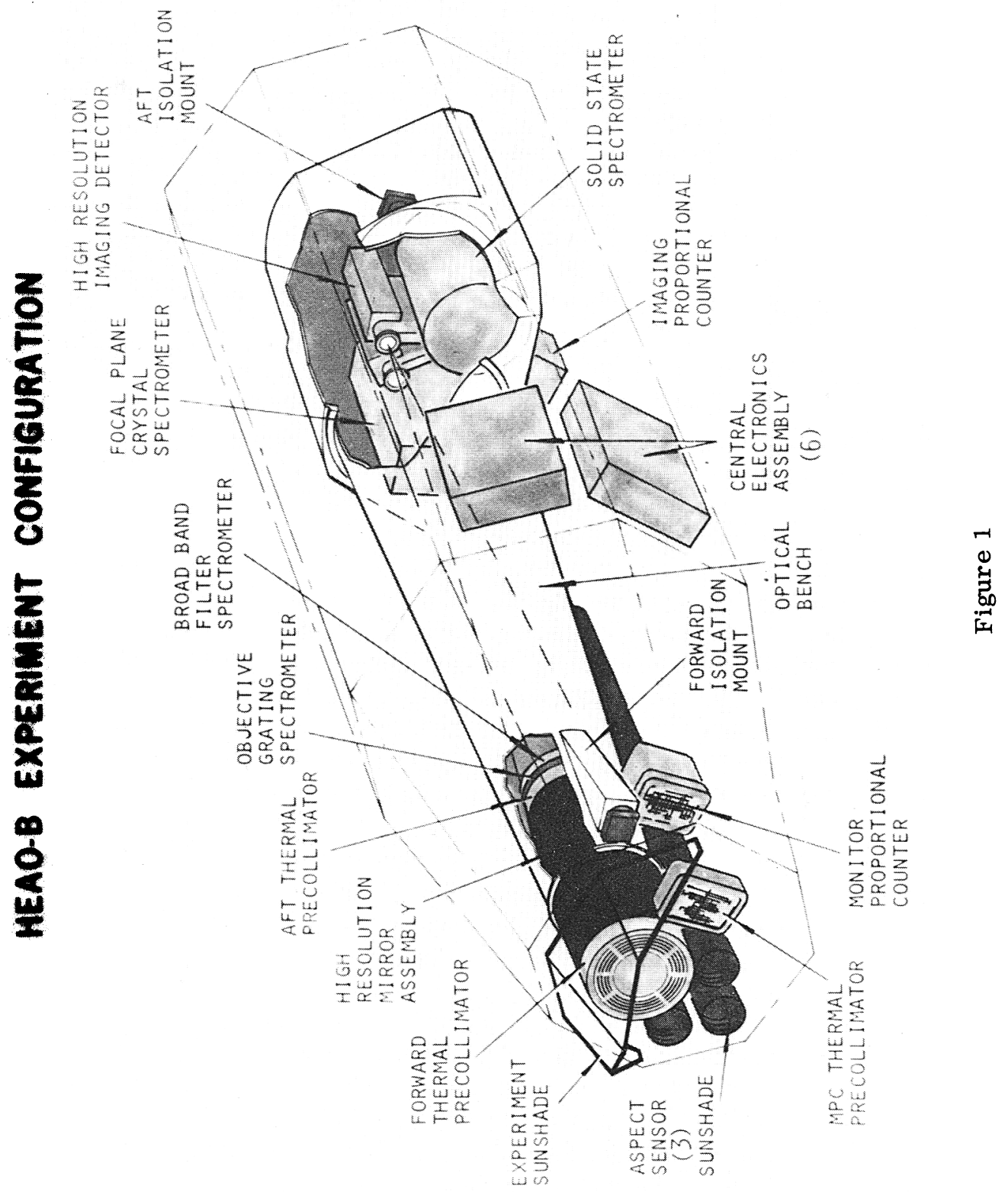


The use of focusing optics results in an increase of sensitivity of a factor of 1000 over any previous surveys and provides an angular resolution of a few arc seconds comparable to that usually achieved in visible light. These new observational capabilities have profoundly changed the scope of Xray astronomy. $\mathrm{X}$-ray emission can now be detected from intrinsically weak sources, such as the coronas of stars $\left(10^{26}-10^{33} \mathrm{erg} \mathrm{sec}-1\right)$ or from the most distant known members of intrinsically bright classes of objects such as clusters of galaxies and QSO's (1043 - $\left.10^{48} \mathrm{erg} \mathrm{sec}^{-1}\right)$. Every known astronomical object (save, perhaps, the planets) can now be studied in $\mathrm{X}$-ray radiation.

The manner in which $\mathrm{X}$-ray observations are carried out has also changed substantially. Although the project was conceived and initiated as a Principal Investigator experiment, and carried out under the scientific guidance of a consortium of institutions (Harvard/Smithsonian Center for Astrophysics, Columbia University, Goddard Space Flight Center and Massachusetts Institute of Technology), the capabilities of the Einstein Observatory have been made available to an ever increasing number of guest observers. For the first six months of the mission, 170 proposals for observations by guest investigators have been approved by a NASA Review Committee and are being carried out. This level of participation by astronomers not directly involved with the hardware construction is comparable to that of any major optical or radio astronomy observatory.

These qualitative changes particularly affect the ability of X-ray astronomy to contribute to the study of objects at cosmological distances. Although data reduction of the more than $10^{8}$ bits of information that we receive daily is occurring in essentially real time, data analysis of the result is still in a preliminary phase. Therefore, I will be able today to present only tentative conclusions, more from the point of view of acquainting you with the nature and import of the observations than in the expectation of completely resolving any of the very difficult and long-standing questions that have been discussed at this meeting. I will limit my remarks to three subjects:

X-ray studies of clusters of galaxies
Detection and study of distant QSO's
Preliminary findings of the deep X-ray surveys

\section{CLUSTERS OF GALAXIES}

Detection of X-ray emission from clusters of galaxies was first accomplished from UHURU in the early $1970^{\prime} \mathrm{s}$. Subsequent satellite investigations from OSO-8, Ariel V, ANS and HEAO-1 discovered a large number of cluster sources $(>50)$ and gave us a qualitative understanding of the main 


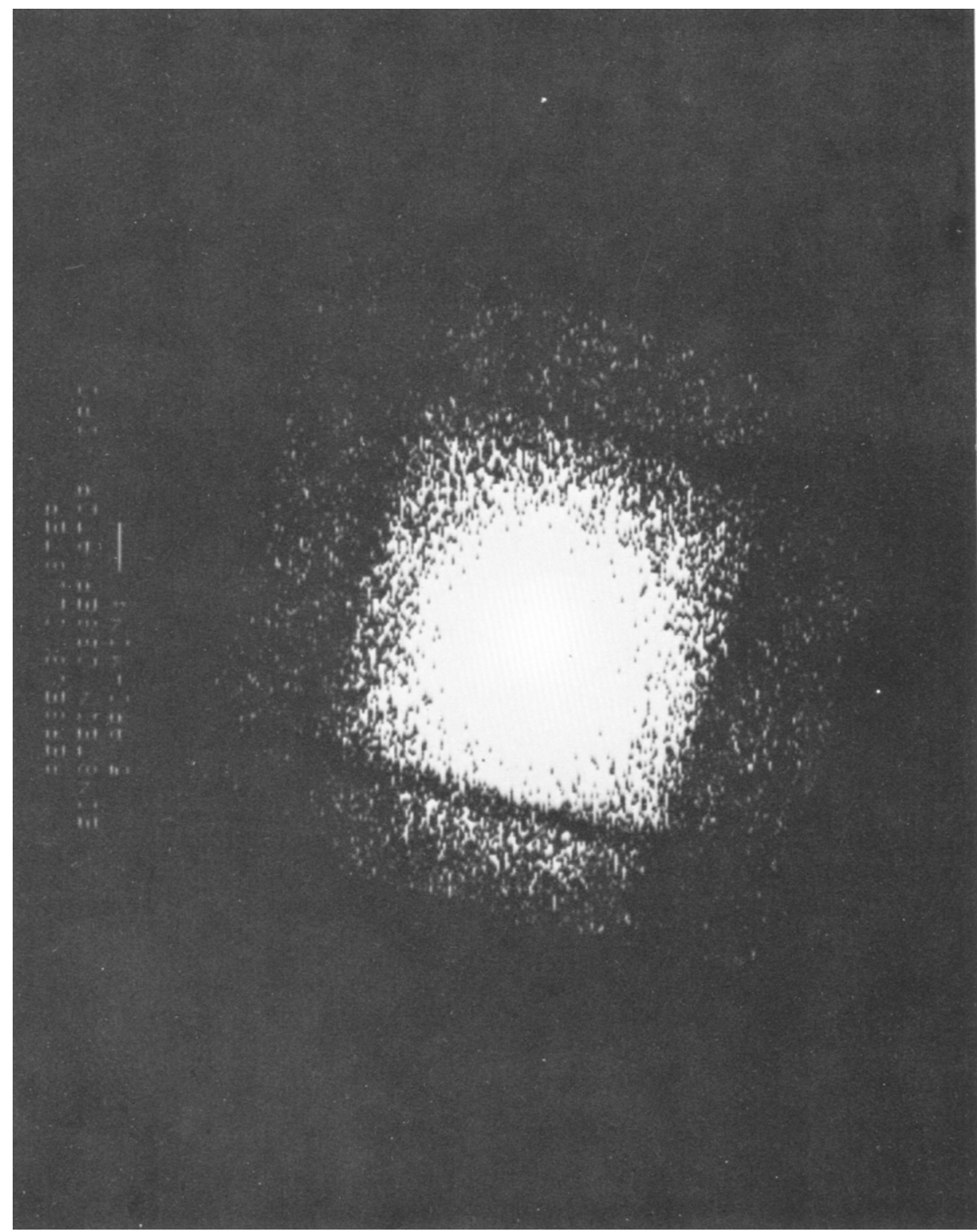

为 

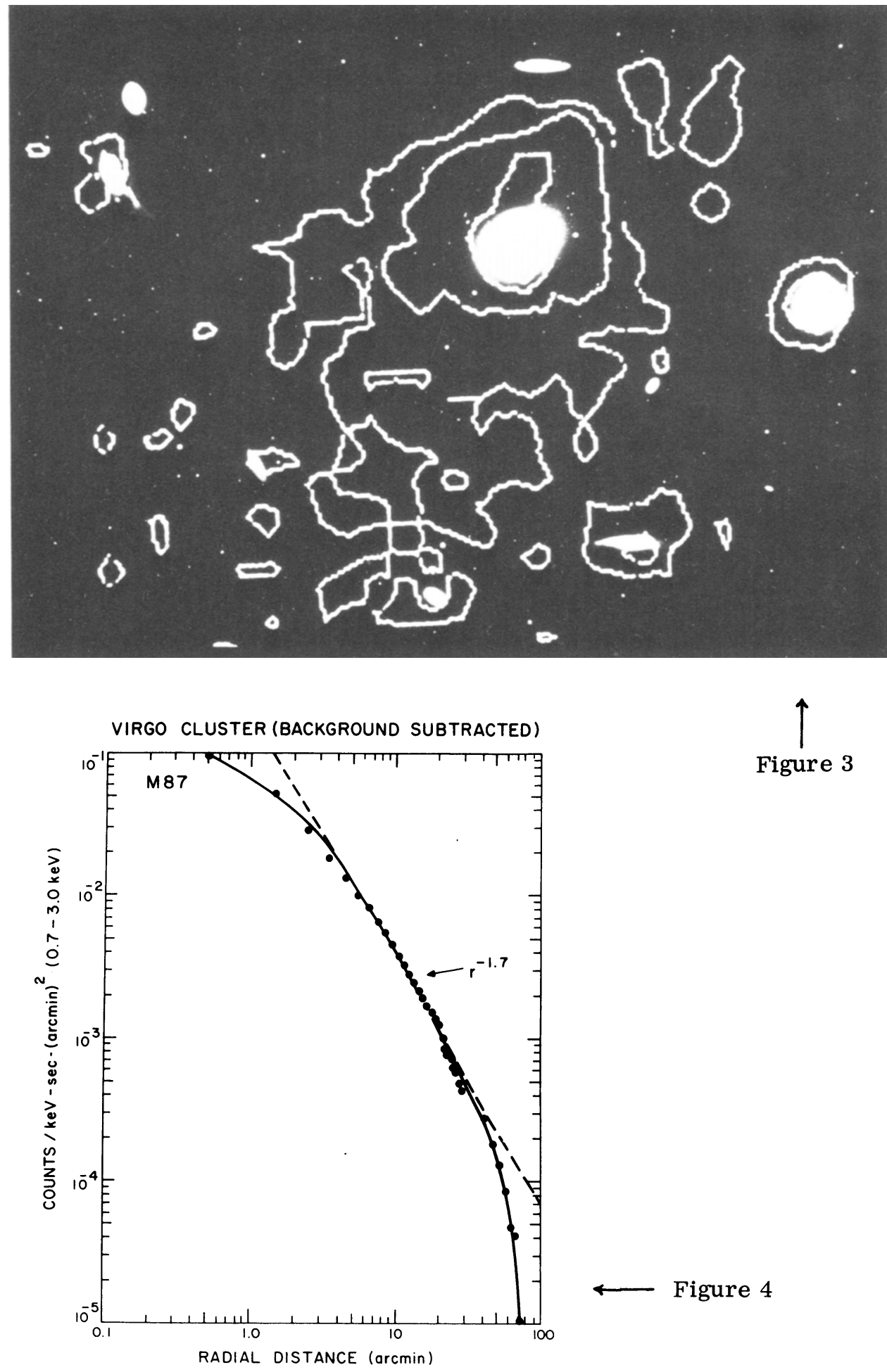

$\longleftarrow$ Figure 4 
features of the physical processes involved. The bulk of the emission originates from a hot intergalactic medium pervading the cluster and containing a total mass comparable to that contained in the visible galaxies. The emission is due to thermal bremsstrahlung and $\mathrm{K}_{\alpha}$ emission lines of iron at $6.7 \mathrm{keV}$ have been detected from several clusters. In some clusters evidence is found for gas with at least two different temperatures. Given the presence of high $\mathrm{Z}$ elements, the gas is believed to be material from the galaxies contained in the cluster rather than primeval gas left over by inefficiencies in the process of formation. Einstein observations contribute to the study of clusters in several distinct areas.

The study of nearby clusters, such as Virgo, Perseus and Coma, can be carried out in some detail. Images of the Virgo field obtained with the IPC, for example, reveal a complex morphology which is being studied at CFA (Forman et al, 1979). The X-ray emission is resolved in several components: emission from M87 itself which is being further studied at high resolution to resolve the nucleus and jet contributions; emission from cool gas ( $2 \mathrm{keV}$ ) surrounding M87 itself, M85 and possibly other galaxies in the cluster; finally, a higher temperature $(\sim 10 \mathrm{keV})$ gas component pervading the entire cluster and presumably following the cluster potential (Figures 2 and 3 ).

Gorenstein and Fabricant, of CFA, have carried out detailed studies on the temperature and source brightness distribution of the gas surrounding M87. In the central region and out to at least 25 arc minutes the emission from the cooler component is predominant and we find essentially a constant temperature of $2.5 \times 10^{7} \mathrm{ok}$. Figure 4 shows the fit to the X-ray data with projection effects not yet removed. Removing projection effects we find that the gas distribution in the region between a few and 25 arc minutes can be well described by a power law $\mathrm{r}^{-1.3}$. If we assume that the gas is in hydrostatic equilibrium, we can derive the underlying mass required to explain the observed distribution. This mass would mainly reside not in the gas $\left(10^{12} \mathrm{M}_{\odot}\right)$, but in a dark halo component such as the one suggested by Bahcall and Sarazin, and by Matthews $\left(10^{13} \mathrm{M}_{\odot}\right)$. We find for this mass a distribution of $\mathrm{r}^{-2}$ and a total mass (within $100 \mathrm{kp}$ ) of $10^{13} \mathrm{M}_{\odot}$. We will be able to apply similar analysis techniques to the study of M86, although at the moment we do not yet have sufficient statistics to go into as much detail. The cool gas component surrounding M86 appears to have a temperature of $\sim 1 \mathrm{keV}$ and central gas density of $4 \times 10^{-3}$ particles/cc. The total mass of the gas within $60 \mathrm{kpc}$ is found to be of order $6 \times 10^{9} \mathrm{M}_{\odot}$.

Since we know from previous experiments, as well as the spectrometer data on Einstein, of the existence of an additional hot component of the gas, we can study the conditions under which M86 will lose its gas halo when crossing the center of the Virgo cluster due to ram pressure stripping. Our data, 


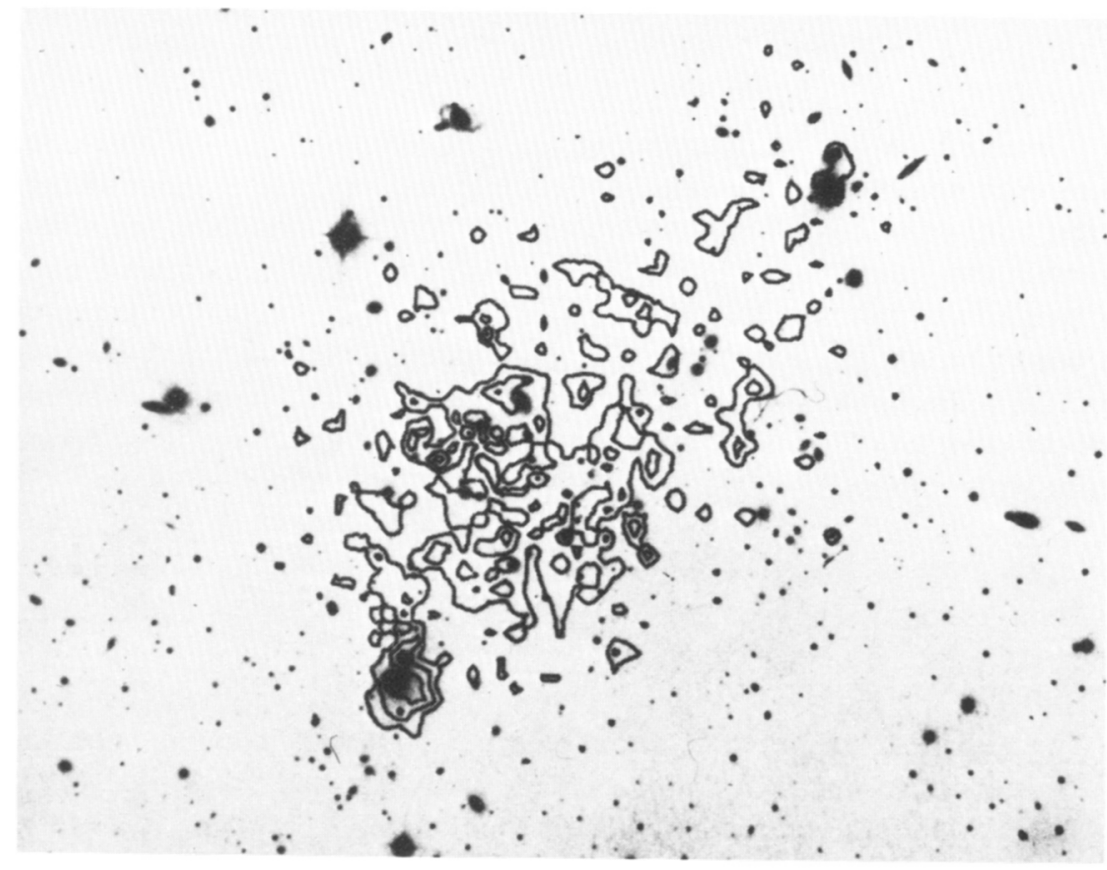

Figure 5

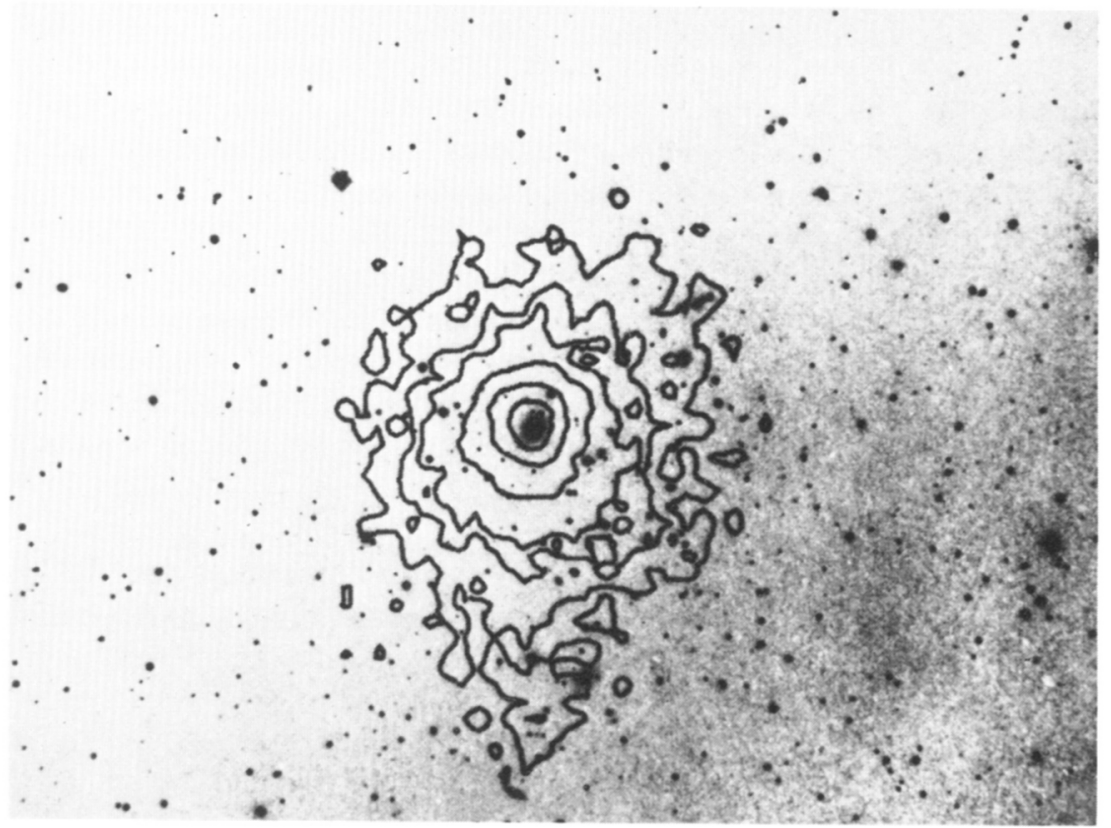

Figure 6 
therefore, strongly suggests that at least some of the gas originated in the galaxies.

Probably M86 is approaching the cluster center now, as indicated by its velocity component towards us; taking into account the densities of the hot and cold gas components, the velocity of the galaxy and its mass one can show that the gas will quite probably be stripped during its next crossing (Forman et al, 1979). Many other sources are detected in Virgo; while some turn out to be simply foreground or background objects, we find that we detect all bright galaxies with $\mathrm{M}_{\mathrm{V}} \Leftarrow 12.5$. These galaxies could either be intrinsic emitters or be surrounded by glowing gas either lost from the galaxy or accreting onto it.

This type of detailed study of cluster properties is being carried out for Coma and Perseus, as well, although the analysis of these clusters is still in its early stage.

In addition, preliminary analysis of spectroscopic data obtained both with the non-dispersive solid state spectrometer and with the Bragg Crystal spectrometer confirms the existence of a two-temperature gas in general agreement with the qualitative description given above. The SSS spectrometer, for instance, resolves lines from both hydrogen and helium like atoms of silicon and sulphur and from their relative strength the temperature of the two components can be measured.

For more distant clusters, such detailed studies cannot yet be carried out. However, the imaging experiment reveals a complex morphology of the gas distribution which is closely related to the cluster morphology and probably to their evolutionary state (Jones et al, 1979). Figures 5 and 6 of A1367 and A85 exemplify two extreme cases of a very clumpy and broad, and a very centrally condensed and smoothly distributed cluster medium. Figure 7 illustrates our findings for a number of clusters. We tentatively identify at least four different types. Two extreme cases mentioned above are an intermediate case, exemplified by A2256, which is centrally enhanced but less peaked than A85, and finally the type exemplified by A2666, a poor group containing a cD galaxy.

Correlation between X-ray properties and morphological type and evolutionary state has been discussed by Jones et al and by N. Bahcall.

In rough outline it is clear that the observed $\mathrm{X}$-ray properties fit well in the context of an evolutionary scheme such as that discussed by Peebles or Perrenod. 


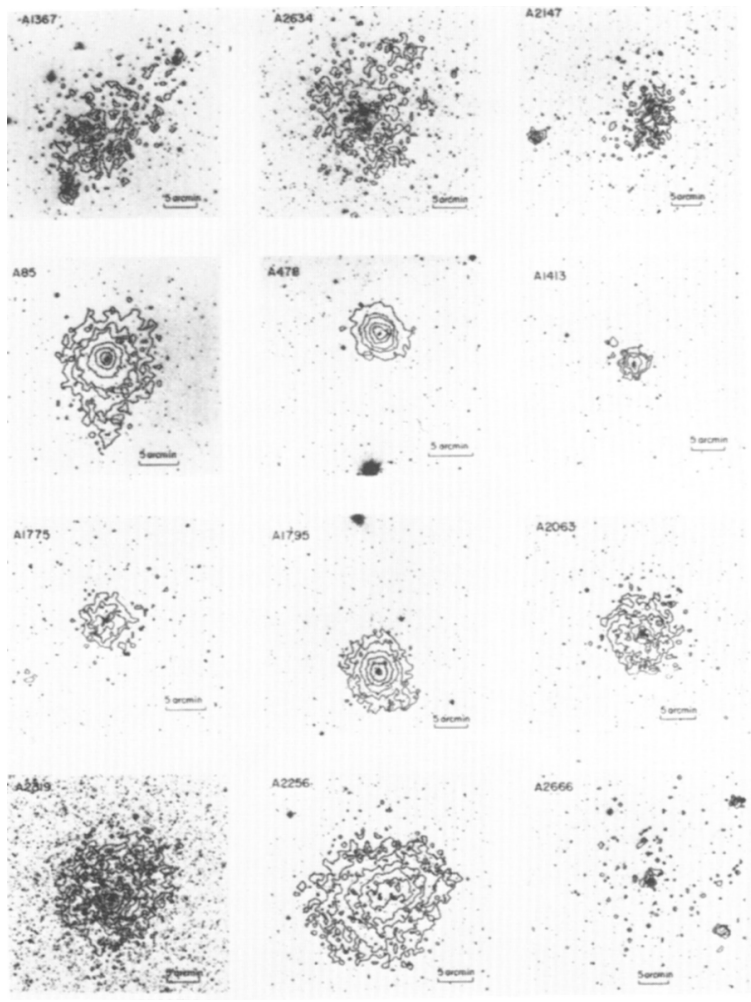

Figure 7

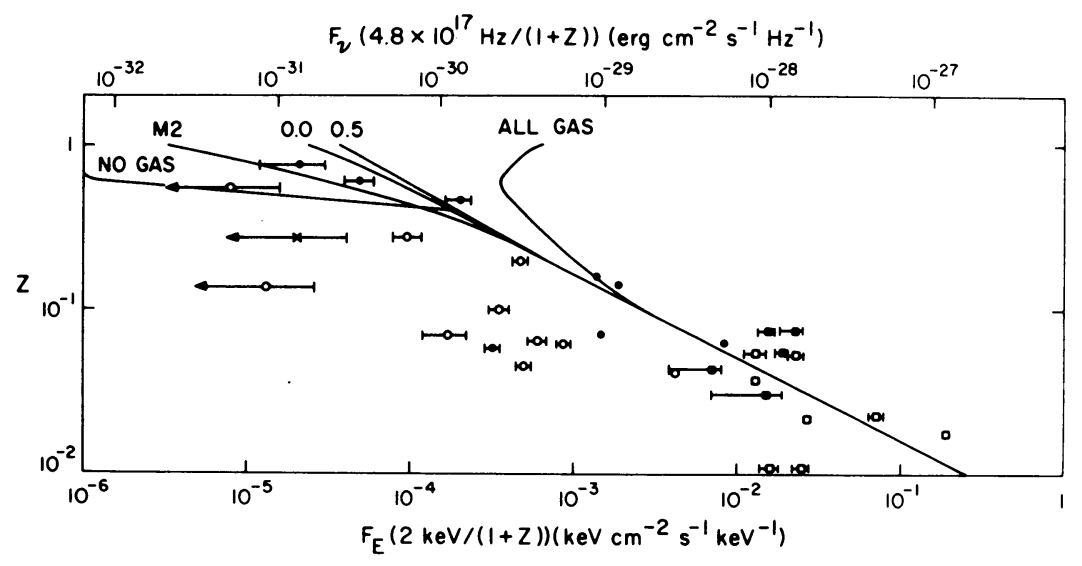

Figure 8 
Time does not permit me today to dwell on this point; I would only like to remark that once the extensive survey of clusters which is planned in the Einstein mission is completed, the X-ray measurements will give us a solid quantitative base to discuss integral properties of clusters. It is clear that the gas distribution can be measured more readily than the galaxy distribution and the temperature of the gas is an easier quantity to measure than the velocity dispersion particularly for distant clusters.

It may at some point become more convenient to carry out searches and classification of clusters on the basis of their X-ray, rather than optical, properties. In this connection, we have extended the detection of clusters to the furthest known objects of this kind with a view toward studying possible evolutionary effects. Figure 8 summarizes our preliminary findings. It is clear that we can readily detect any cluster discovered in visible light observation. At the moment the large intrinsic luminosity dispersion and the presence of what are understood as different evolutionary stages at any given epoch makes the interpretation of the results extremely complex. Notwithstanding these problems which hopefully will be resolved by additional data, we seem to be able at least to discriminate against the most extreme hypothesis of cluster evolution.

I would like to make clear that although we have described the data within the simple evolutionary scheme, for instance of Perrenod, the data are yet too incomplete to rule out competing models. In fact, there already are indications that not all may be well in the simple evolutionary picture which I have used as a framework to describe the data. The X-ray detection of 3C295, indicating that there is sufficient intergalactic gas to cause stripping of the cluster spirals, although the colors of these galaxies imply that they have not been stripped, is, for instance, such an indication (Dressler, 1978).

In fact, we are just beginning the X-ray study of clusters in some detail and it may well be that our views of cluster formation and evolution will have to be considerably modified, as a result of these observations.

For the future, X-ray research promises to be a very powerful tool for cosmology. As an example, it is sufficient to mention the recent proposal for an absolute measurement of $\mathrm{H}_{\mathrm{O}}$ and $\mathrm{q}_{\mathrm{Q}}$ through measurement of temperature and of X-ray surface brightness distribution in clusters and cooling of the microwave radiation (Zeldovich-Sunyaev effect) made independently by several authors. This research is currently in progress, but the main difficulty is the scarcity of reliable data in the microwave domain. 


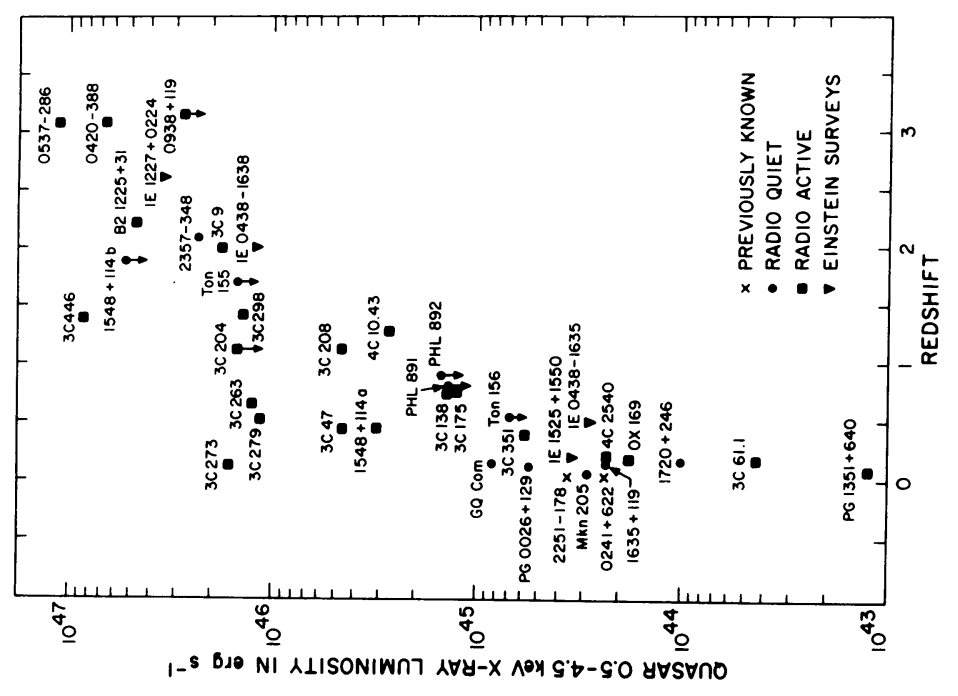

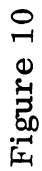

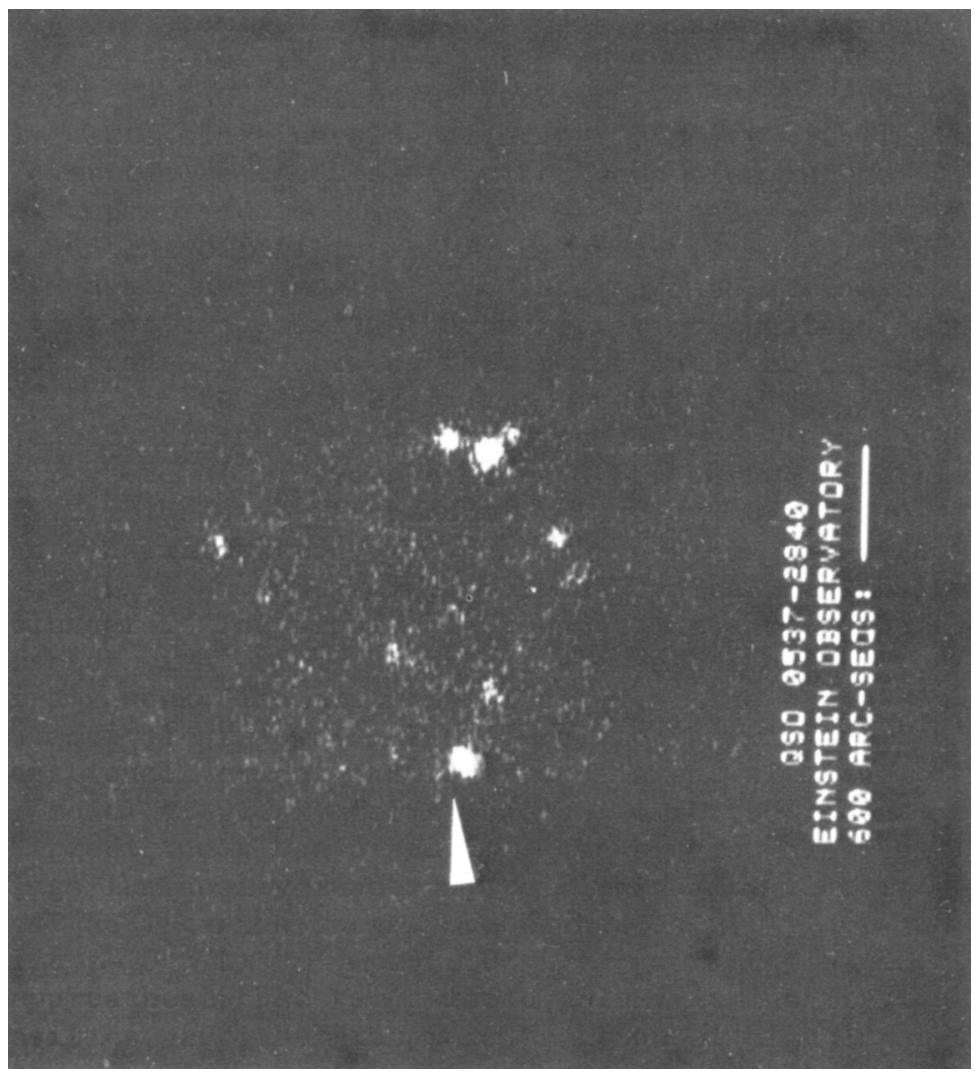

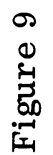


QUASARS

We are carrying out a program of observations to detect $\mathrm{X}$-ray emission from known QSO's which have been selected to span a wide variety of optical and radio properties and also a significant range of redshifts (Tananbaum et al, 1979). Prior to Einstein, only a few quasars had been detected as X-ray sources, the furthest at redshift $\mathrm{z}<0.2$. We have now observed over 40 QSO's with the furthest at redshift $z=3.1$. In addition, more than 20 new quasars have been discovered with redshifts ranging from 0.5 to 2.1 as a result of deep surveys and optical follow-ups.

Figure 9 shows the results of an observation of the QSO 0537-286 which was obtained with the IPC after a 10,000 second exposure. The quasar is indicated by the cursor and has a luminosity of $10^{47} \mathrm{erg} \mathrm{s}^{-1}$ in the 0.5 to 4.5 $\mathrm{keV}$ band (at the source and assuming $\mathrm{q}_{0} \approx 0$ ). As can be seen, there are several sources detected in this field $\left(\sim 1^{0} \times 1^{0}\right)$. These include two main sequence $F$ stars and several as yet unidentified sources which are fainter than 15th magnitude. The ease with which we have observed this quasar, and a second quasar (0420-388) also at $\mathrm{z}=3.1$ indicates that with the Einstein Observatory we should be able to detect still more distant QSO's. Thus, the cosmologically intriguing question of the formation epoch for QSO's may be within our ability to answer. Of course this depends upon assumptions regarding evolution of the quasars (both in density and luminosity) which may be present.

A summary of the X-ray properties of QSO's is shown in Figure 10. This is a plot of the 0.5 to $4.5 \mathrm{keV}$ luminosity (at the source) versus redshift. The luminosity range is from $10^{44} \mathrm{erg} \mathrm{cm}^{-2} \mathrm{~s}^{-1}$ to $10^{47} \mathrm{erg} \mathrm{cm}^{-2} \mathrm{~s}^{-1}$. We observe that there is not obvious redshift-luminosity correlation. The absence of low luminosity points at high redshifts is due to selection effects since the sensitivity of the observations is limited. Radio quiet and radio bright quasars are indicated by symbols. Thus far we have observed primarily radio bright QSO's. This may be a source of bias in any preliminary conclusions which may be drawn from the data. However, those radio quiet quasars we do observe, do not appear to be significantly different in their X-ray properties , suggesting little, if any, correlation of $\mathrm{X}$-ray and radio properties.

Tananbaum et al (1979) have found a tentative relation between the optical and X-ray fluxes of quasars such that the slope of a power law joining the X-ray and optical flux densities is nearly constant with average slope $\alpha_{\text {ox }} \approx 1.30$. This is illustrated in Figure 11 where $\alpha_{0 x}$ is plotted for each of the observed QSO's. Most significant is that $\alpha_{\text {ox }}$ shows no apparent dependence on redshift and again there is no evidence for a bias with regard to the quasar radio properties. Using the optical luminosity function for QSO's from Braccesi et al (1979) and the value of $\alpha_{\text {ox }} \simeq 1.30$, the contribution of QSO's to the X-ray 


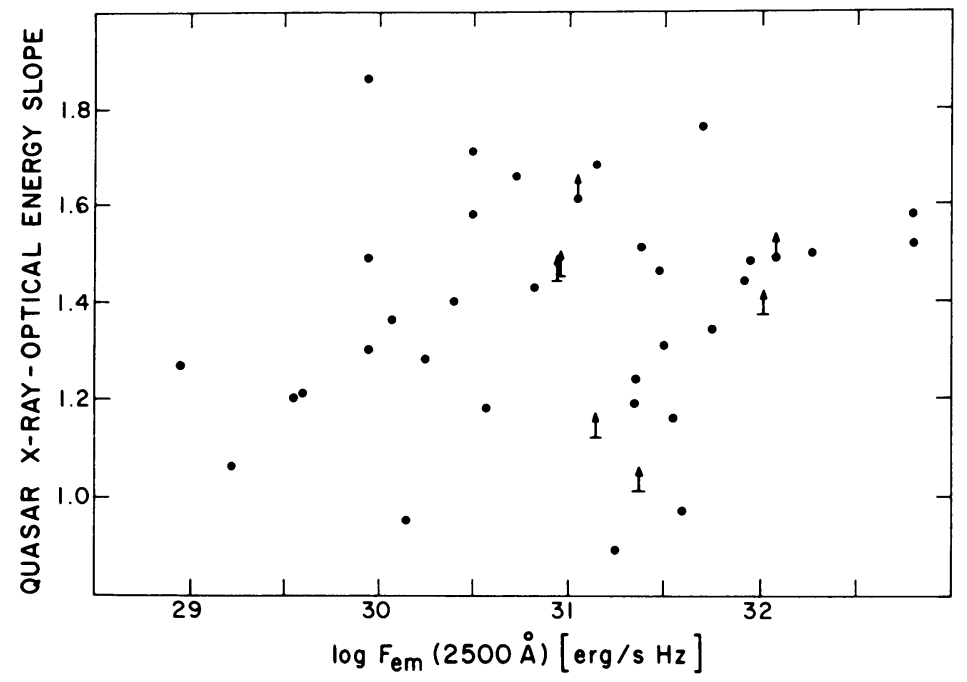

Figure 11

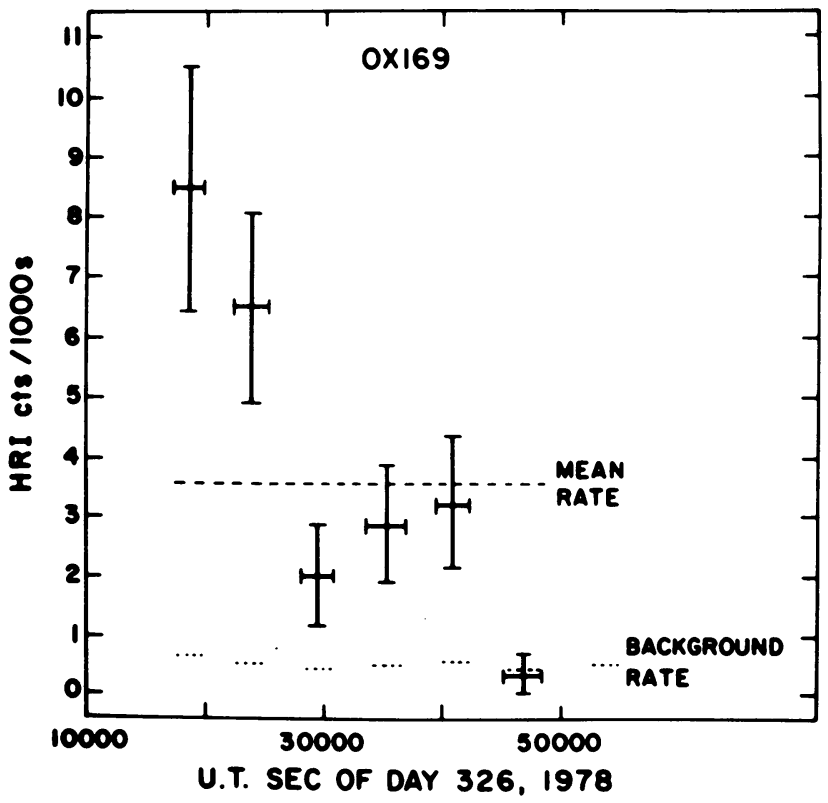

Figure 12 
background can be calculated. The results of such a calculation depend strongly on the value of $\alpha_{\mathrm{ox}}$ and the range of QSO luminosity which Tananbaum et al have taken as $10^{44}$ to $10^{46} \mathrm{erg} \mathrm{s}^{-1}$. They find that from $10 \%$ to all of the $\mathrm{X}$-ray background can be accounted for from quasars. Moreover, for some choices of the luminosity function and the evolution of quasars, that is, the value of $\mathrm{n}$ and $\mathrm{z}_{\text {max }}$ in the density evolution function $(\mathrm{z}) \alpha(1+\mathrm{z})^{\mathrm{n}}$ and $\mathrm{z} \leq \mathrm{z}_{\max }$, the X-ray background is exceeded. Thus the X-ray data can be used to place constraints on quasar evolution. For example, current observations of quasars are not consistent with a local origin hypothesis unless they are at least $500 \mathrm{Mpc}$ away, which is then essentially equivalent to a cosmological interpretation of the redshifts. This conclusion is based on the arguments originally made by Setti and Wolter (1973).

The emission mechanism for QSO's is a major astrophysical problem since we are concerned with processes capable of providing luminosities of up to $10^{47} \mathrm{erg} \mathrm{s}^{-1}$. X-ray studies of quasars may provide unique insights into this question by studying time variability of QSO emission. Figure 12 shows the X-ray observations of QSO OX169 which is at redshift $\mathrm{z}=0.2$. The source is variable with a decrease in luminosity from 2.0 to $0.6 \times 10^{44}$ erg $\mathrm{s}^{-1}$ within 100 minutes. This behavior can be interpreted as a signature of the underlying energy source of the quasar. For a process involving conversion of mass to energy (which seems likely on the basis of the large amounts of energy involved), rapid variability requires high efficiency. This supports models involving release of gravitational energy through accretion onto a compact object. If such a model for OX169 is assumed, then a black hole with mass between $10^{6}$ to $10^{8} \mathrm{M}_{\Theta}$ is required so that neither the Eddington luminosity limit, nor the size scale from variability, is exceeded. We also note that the short time scale requires the X-rays to be produced near the central source, which implies that $\mathrm{X}$-ray observations may ultimately provide the means for understanding mechanisms for powering quasars and active galaxies in general.

\section{DEEP X-RAY SURVEY}

The increased sensitivity of the Einstein Observatory has made possible for the first time a truly deep survey in limited areas of the sky. Of primary concern in conducting these surveys has been the long-standing question of the origins of the X-ray background. Both diffuse and discrete models have been proposed and are discussed extensively in the literature (cf. a recent review by Schwartz, 1978). We can approach the problem directly with Einstein observations through deep surveys by attempting to image the background at the limit of sensitivity. The expected source density, if the background is discrete, is high enough so that the 2 arc min resolution of the IPC is near the source confusion limit. The HRI, of course, is virtually free from this problem. 
Without going into details of our observing program, which is described in the deep survey paper by Giacconi et al (1979b), I will present the results which are already of substantial significance. Figure 13 shows an example of the observing pattern for a deep survey. This is for the region in the constellation Eridanus. We have conducted a similar survey in the constellation Draco. Observing times vary from 10,000 to 50,000 seconds. The flux limit of the survey is $1.3 \times 10^{-14} \mathrm{erg} \mathrm{cm}^{-2} \mathrm{~s}^{-1}$ or about $10^{-7} \mathrm{Sco} \mathrm{X}-1$. In the two surveys thus far completed, we detect $43 \mathrm{X}$-ray sources in about $1 / 2$ square degree of sky. Our positional accuracy in locating sources is dependent on the detector used and the source intensity. In the case of the IPC we have adopted a positional uncertainty of 60 arc sec radius mainly due to systematic uncertainties in converting detector coordinates to sky positions. For the HRI sources the location accuracy varies from $\sim 5$ to $\sim 20$ arc sec radius, which depends mainly on the statistical uncertainty from the small number of source counts.

Since we are concerned with the contribution of discrete sources to the extragalactic X-ray background, we first limit our band to the 1 to $3 \mathrm{keV}$ range (for conversions of count rate to flux we assume that the sources have the spectrum of the background) and then we separate the contribution of stars from the objects detected. This is done by direct measurement of the redshifts of candidate objects where possible, or by the optical and radio morphology of potential counterparts. To accomplish this we have been assisted by various optical and radio astronomers whose contributions to our study are greatly appreciated. We have also measured the $\mathrm{B}$ magnitude and $\mathrm{B}-\mathrm{V}$ colors for optical candidates as well as their proper motions over a 25-year baseline. In making these observations we have identified new quasars, compact galaxies, and possible BL Lac objects. In some instances we have found X-ray error boxes which appear completely empty at the plate limit of available optical material. In one such case additional observations using CCD cameras at Mt. Hopkins (Gursky and Schild, 1979) and Mt. Palomar (Kristian, Westphal and Young, 1979) have revealed the presence of objects fainter than $M_{R} \sim 21.5$. We conclude that about $1 / 3$ of the $\mathrm{X}$-ray sources are associated with stars and we eliminate these from further consideration.

Of the remaining source we select, for the purposes of estimating the background contribution, only those within the central $32 \times 32$ arc min region of the survey field and which are observed at the 5 sigma level of significance or higher. These constraints reduce possible errors due to statistical uncertainty in source intensity and geometrical corrections to the flux. The results are shown in Figure 14 which is a plot of the number-flux observations for $\mathrm{X}$-ray sources. Included in this figure are the previous distributions from the UHURU survey (Murray, 1977), and the HEAO-1 flux limit, our new result and the Einstein flux limit for an exposure of $5 \times 10^{5}$ seconds. We also indicate the background limit for a number-flux relation which has the form $N(>S) S^{-1.5}$. 

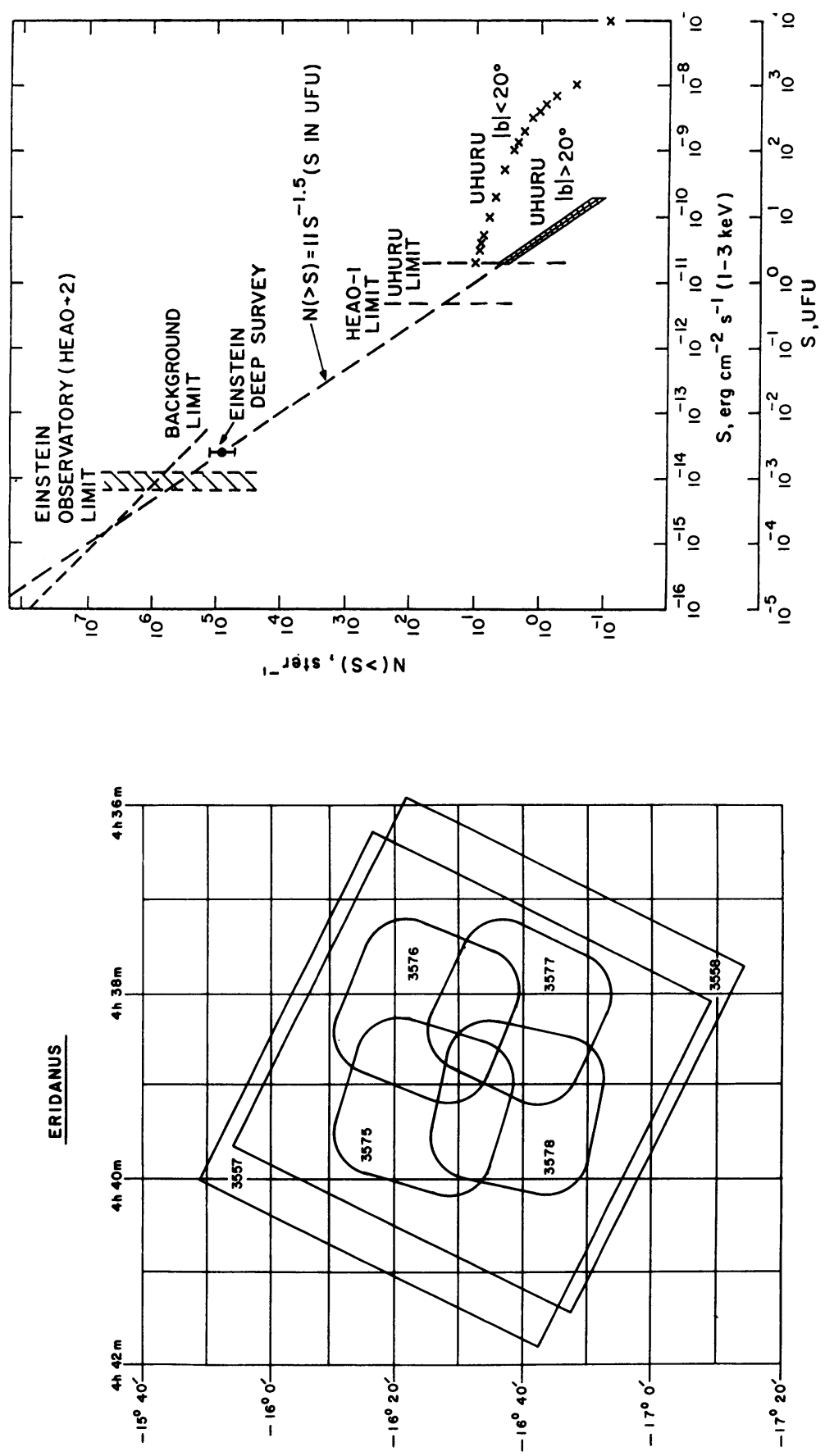

我 
The Einstein deep survey point is at a flux of $2.6 \times 10^{-14} \mathrm{erg} \mathrm{cm}^{-2} \mathrm{~s}^{-1}$ and corresponds to $6.3 \times 10^{4}$ sources ster ${ }^{-1}$. This lies just below an extrapolation of the UHURU results over almost 3 orders of magnitude in flux. This agreement indicates that this simple form for $N(P)$ is valid over a large dynamic range in flux. This can be explained either in terms of a uniform distribution of objects in space which would have to be nearby, or in terms of cosmological density evolution compensating for cosmological distance-volume effects. This first case would occur if we are detecting only objects within a small range of redshifts and a large range of intrinsic luminosities. This is unlikely in view of the optical data and the general considerations of Schwartz (1978). The second occurs if we are observing sources at large redshift $(z>1)$ as indicated by the optical data. For example, the number-flux relationship for active galaxies, QSO's (assuming a strong density evolution) and nearby classes of X-ray sources, such as rich clusters, can approximate the observed power law slope of 1.5 over our flux interval. In this case we would expect the slope of the number-flux relationship to begin steepening at or beyond the present flux limit as quasars dominate the sources.

Taking the number-flux data point from the deep survey, and assuming a power law index of 1.5 , we compute the contribution of discrete sources to the total extragalactic background to be $(25 \pm 11) \%$ at the flux limit of $2.6 \mathrm{x}$ $10^{-14} \mathrm{erg} \mathrm{cm}^{-2} \mathrm{~s}^{-1}$. An extrapolation of a factor of two in flux is made based on our observation of sources to this faint level. This will give a contribution of $37 \%$ of the background. Even this value is conservative since in selecting sources to include in the calculation of the number-flux relation we have not included sources with observed flux greater than $2.6 \times 10^{-14} \mathrm{erg} \mathrm{cm}^{-2} \mathrm{~s}^{-1}$ if they were below the 5 sigma significance level. Thus, real sources have been left out of the computation. This may be as much as a $30 \%$ effect, which is an underestimation of the source counts. Thus, we feel quite confident in concluding that a substantial fraction, if not all, of the extragalactic X-ray background is due to discrete sources. We note that while previous theoretical discussions of the contribution of discrete sources to the background have yielded estimates comparable to our result, they were based on an extrapolation of at least 3 orders of magnitude in flux. The Einstein deep surveys have given us the first direct observation of these sources at fluxes low enough to yield a substantial contribution.

\section{CONCLUSION}

The Einstein X-ray Observatory has given X-ray astronomy an observational capacity not too different from that available in the optical and radio wavelengths, especially for the study of objects at cosmological distances. $\mathrm{X}$-ray observations have unique capabilities in the study of high energy processes and for probing the most distant reaches of the Universe. Observations with 
Einstein have already shed new light on the distribution of quasars and their contribution to the X-ray background. Studies of variability should prove to be a powerful tool in understanding the underlying energy source for these objects. The X-ray study of clusters promises to be extremely interesting, even on the basis of our preliminary results. The deep survey has extended our knowledge of X-ray sources to the point where much of the X-ray background is resolved. We have reached a sensitivity which allows us to begin to observe cosmological and evolutionary effects.

\section{REFERENCES}

Bahcall, J. and Sarazin, C.L., 1978 Ap. J. 219, 781.

Bahcall, N. 1979 Joint Discussion on Extragalactic High Energy Astrophysics IAU Montreal - to be published.

Braccesi, A., Zitelli, V., Bonoli, F., and Formiggini, L. 1979 submitted to Astronomy and Astrophysics.

Dressler, A., 1978 Ap. J. 226, 55, 113-E6.

Fabricant, D., Lecar, M., and Gorenstein, P. , 1979 Joint Discussion on Extragalactic High Energy Astrophysics, IAU Montreal - to be published.

Forman, W., Schwarz, J., Jones, C., et al, 1979 Ap. J. (Letters) in press Giacconi, R., Branduardi, G., Briel, U. et al, 1979a Ap. J. 230: 540-550. Giacconi, R., Bechtold, J., Branduardi, G. et al, 1979b Ap. J.(Letters) in press

Gursky, H. and Schild, R., 1979. Private communication Jones, C., Mandel, E., Schwarz, J., et al 1979 Ap. J. (Letters) in press Kristian, J., Westphal, J., and Young, P. 1979. Private communication Mathews, W. G., 1978 Ap. J. 216, 413.

Murray, S. S. 1977, CFA Preprint \#680.

Peebles, P.J.E. 1970 A. J. , 75, 13.

Perrenod, S. 1978 Ap. J. 226, 566.

Schwartz, D.A. 1979 COSPAR X-RAY ASTRONOMY, edited by W. A. Baity and L. E. Peterson; Pergamon Press Oxford and New York 1979.

Setti, G. and Woltjer, L., 1973, "X-and Gamma-Ray Astronomy", IAU Symposium No. 55 (eds. H. Bradt and R. Giacconi); D. Reidel Publishing Co.

T ananbaum, H., Avni, Y., Branduardi, G., et al. 1979 Ap. J。 (Letters) in press. 


\section{DISCUSSION}

Perrenod: How many square degrees have your surveys covered? I take it that you've seen no clusters.

Giacconi: We have not yet detected clusters in the deep surveys. They only covered less than $1^{\circ}$ of the sky.

Silk: The clusters that exhibit the Butcher-Oemler effect appear to show a radial gradient in the color distribution of the galaxies, with the bluer galaxies being preferentially concentrated in the center regions. Have you made a detailed comparison of the extent of the $\mathrm{X}$-ray emitting region with the blue galaxy distribution? This is evidently necessary before one can decide on the relevance of ram pressure stripping models for these clusters.

Giacconi: We have not yet analyzed the results from that point of view.

Stockton: You showed the X-ray variations in $0 \mathrm{X}-169$ and mentioned that 3C 273 also showed variations at about 10\%. These two QSO's are the only two I know of that show bright optical jets. Have you looked for variations in other QSO's?

Giacconi: Yes, but we haven't found any.

Creen: Has any correlation been found between X-ray (not X-ray optical) spectral index and redshift in quasars?

Giacconi: Not yet; we are studying the question.

Peebles: Can you use the X-ray luminosities of the main sequence $M$ stars to place limits on the hypothesis that galaxies have massive halos of low mass main sequence stars?

Giacconi: A galaxy containing $10^{11}$ to $10^{12}$ M-type stars will produce 1038 to 1040 ergs per sec in X-rays. Massive halos from nearby galaxies could therefore be detected. Conversely, lack of detection may be useful in setting upper limits on the number density of M-stars.

Abel2: Following up on Jim Peebles' question, how many M dwarfs have now been observed for $\mathrm{X}$-ray emission, and what fraction of them show the surprising ratio of $\mathrm{L}_{\mathrm{X}} / \mathrm{L}_{\mathrm{V}}$ of $10^{-1}$ ?

Giacconi: About a dozen M dwarfs have been observed; most of them show this ratio. 